\title{
Abundancia de peces en los complejos arrecifales de Serranilla, Bajo Alicia y Bajo Nuevo, Reserva de Biósfera Seaflower de Colombia
}

\author{
Fish abundance in the reef complex of Serranilla, Alice Shoal \\ and New Shoal, Seaflower Biosphere Reserve of Colombia
}

\author{
Heins Bent Hooker ${ }^{*}$, Alfredo Abril-Howard ${ }^{1}$, Nacor Bolaños Cubillos ${ }^{1}$ y Elizabeth Taylor ${ }^{l}$
}

\begin{abstract}
RESUMEN
Entre marzo y abril de 2010 se realizó la expedición científica de recolección de datos biológicos en zonas remotas de la Reserva de Biósfera (RB) Seaflower (complejos arrecifales de Serranilla, Bajo Alicia y Bajo Nuevo). La información recolectada correspondió a abundancia, riqueza y porcentaje de cobertura de comunidades coralinas, ícticas, macroinvertebrados, tortugas, plancton, entre otras especies. Este estudio presenta los resultados de las abundancias en las comunidades ícticas, evaluadas en 82 estaciones (26 en Serranilla, 14 en Bajo Alicia y 42 en Bajo Nuevo). El muestreo evidenció que en Serranilla la especie más importante en el intervalo de tallas de 5-10 cm fue Stegastes partitus, en 11-20 y 21-30 cm Haemulon album, en 31-40 cm Scarus vetula, y mayor de $50 \mathrm{~cm}$ Haemulon melanurum; en Bajo Alicia, la especie más importante en el intervalo de tallas de 5-10 cm fue $S$. partitus, en 11-20 cm Caranx ruber, en 21-30 y 31-40 cm B. vetula, y mayor de $50 \mathrm{~cm}$ Sphyraena barracuda. Finalmente, en Bajo Nuevo, la especie más importante en el rango de tallas de 5-10 cm fue A. coeruleus, en 11-20 cm Scarus taeniopterus, en 21-30 cm Mellichthys niger, en 31-40 cm H. album, en 40-50 cm Balistes vetula, y mayor de $50 \mathrm{~cm}$ Gynglimostoma cirratum, cuya densidad es superior a lo reportado en otras regiones de la RB Seaflower. Como conclusión de los resultados obtenidos y como función de RB Seaflower, se deben aplicar medidas de protección de las especies de importancia pesquera y de toda la diversidad de peces arrecifales presentes en el Archipiélago.
\end{abstract}

Palabras claves: Abundancias, peces arrecifales, interés ecológico y económico, rangos de tallas, RB Seaflower.

\begin{abstract}
During the months of March and April 2010, biological data was collected in the remote areas of the Seaflower Biosphere Reserve (BR) (reef complex including Serranilla, Alice Shoal, and New Shoal). Data included coral communities such as fish (richness and abundance of fish), macro-invertebrates, turtles, plankton, and other regional species. This study shows the results of abundance of fish communities, which were evaluated in 82 sampling areas (26 in Serranilla, 14 in Alice Shoal, and 42 in New Shoal), in order to seach for information about the abundance of species with ecological and economic value by size ranges. Sampling showed that in Serranilla the most important species in the $5-10 \mathrm{~cm}$ size range was $S$. partitus; in the $11-20$ and $21-30 \mathrm{~cm}$ size intervals was Haemulon album; in the 31-40 cm range was Scarus vetula while above $50 \mathrm{~cm}$ was Haemulon melanurum. In Alice Shoal the most important species in the 5-10 cm size range was $S$. partitus; in the 11-20 cm range was Caranx ruber; in the 21-30 and 31-40 cm range was B. vetula while in more than $50 \mathrm{~cm}$ was Sphyraena barracuda. Finally, in New Shoal, the most important species in the $5-10 \mathrm{~cm}$ size range was $A$. coeruleus; in the $11-20 \mathrm{~cm}$ range was $S$. taeniopterus; in 21-30 $\mathrm{cm}$ was Mellichthys niger; in 31-40 $\mathrm{cm}$ was $H$. album; in $40-50 \mathrm{~cm}$ was B. vetula, and above $50 \mathrm{~cm}$ was Gynglimostoma cirratum, whose density was much higher than was reported in other regions of the Seaflower BR.
\end{abstract}

Keywords: Abundance, reef fish, economic and ecological value, size ranges, Seaflower BR.

1 Corporación Ambiental para el Desarrollo Sostenible del Archipiélago de San Andrés, Providencia y Santa Catalina (CORALINA). benthoo@hotmail.com*, alfredoabrilhoward@hotmail.com,nwbc@yahoo.com, elizabeth.taylor@ coralina.gov.co

Recibido 30-IV-2011

Aceptado 31-VII-2012

DOI: http://dx.doi.org/10.15359/revmar.4.2 


\section{INTRODUCCIÓN}

La región territorial de Colombia en el mar Caribe se extiende por 532162 $\mathrm{km}^{2}$ (Posada et al. 2009), de los cuales la Reserva de Biósfera (RB) Seaflower tiene un área aproximada de $180000 \mathrm{~km}^{2}$ (SIGCORALINA, 2011), es decir, que representa cerca del $34 \%$ del mar territorial en el Caribe colombiano, y en esta zona se encuentra más del $77 \%$ de la extensión total de las áreas coralinas de Colombia (Rodríguez-Ramírez et al. 2005), por lo tanto, son de gran relevancia nacional.

En el proceso de establecimiento del AMP Seaflower se realizaron expediciones científicas a siete de los atolones de la RB Seaflower en un esfuerzo conjunto entre CORALINA, la Gobernación Departamental y otras instituciones como The Ocean Conservancy y la Universidad Nacional de Colombia, entre los años 2001-2003, 2009 y 2010-2011, durante los cuales se realizaron monitoreos en el Área Marina Protegida del Norte (atolones de Serrana, Roncador y Quitasueño), Centro (islas de Providencia y Santa Catalina) y Sur (isla de San Andrés y los atolones de Bolívar y Albuquerque), descritos en los trabajos de Dahlgren et al. (2003), Heinemann et al. (2004), Herrón (2004), Sánchez et al. (2005) y los informes técnicos de CORALINA de los años 2009 y 2010. Sin embargo, estos estudios han sido puntuales o parciales, considerando las limitaciones logísticas y financieras que representa trabajar en zonas lejanas y oceánicas; por lo tanto, el desarrollo de este estudio reviste una gran importancia en el avance del conocimiento de la biodiversidad, al obtener información de 82 estaciones en nuevas áreas que están siendo estudiadas por primera vez en la RB Seaflower.

\section{METODOLOGÍA}

\section{Área de estudio}

El Archipiélago de San Andrés, Providencia y Santa Catalina comprende un conjunto de islas oceánicas, atolones y bancos coralinos alineados en dirección NNE a lo largo de la Elevación de Nicaragua, tiene una extensión total de 57 $\mathrm{km}^{2}$ de porción terrestre y $250000 \mathrm{~km}^{2}$ de área marina y está conformado por tres islas habitadas, San Andrés, Providencia y Santa Catalina y ocho cayos o bancos coralinos (IGAC, 1986) (Fig. 1); los cayos del norte están comprendidos por seis atolones que son: Serrana, Roncador, Serranilla, Quitasueño, Bajo Nuevo y Bajo Alicia. Los dos cayos restantes son los del sur, conformados por los atolones de Bolívar (Courtdown o ESE keys) y Albuquerque (SSW keys) (Fig. 1).

\section{Monitoreo}

Los censos de abundancia de peces en los monitoreos de Evaluaciones Rápidas de Arrecifes en la zona externa del AMP Seaflower se realizaron en abril de 2010, en el marco de la expedición científica de distribución y abundancia del caracol pala y comunidades coralinas, financiada por la Gobernación Departamental y la Corporación CORALINA. Las estaciones a muestrear se seleccionaron empleando el Sistema de Información Geográfica, por el personal técnico marino y otros miembros de CORALINA, conocedores de los ambientes marinos de las islas. La selección fue aleatoria tratando de cubrir la totalidad de la extensión de las áreas arrecifales y los diferentes ambientes que se presentan. El muestreo lo llevó a cabo el mismo equipo de trabajo, con el fin de que no variaran los criterios de identificación y así evitar sesgos de observador a observador. Cada 


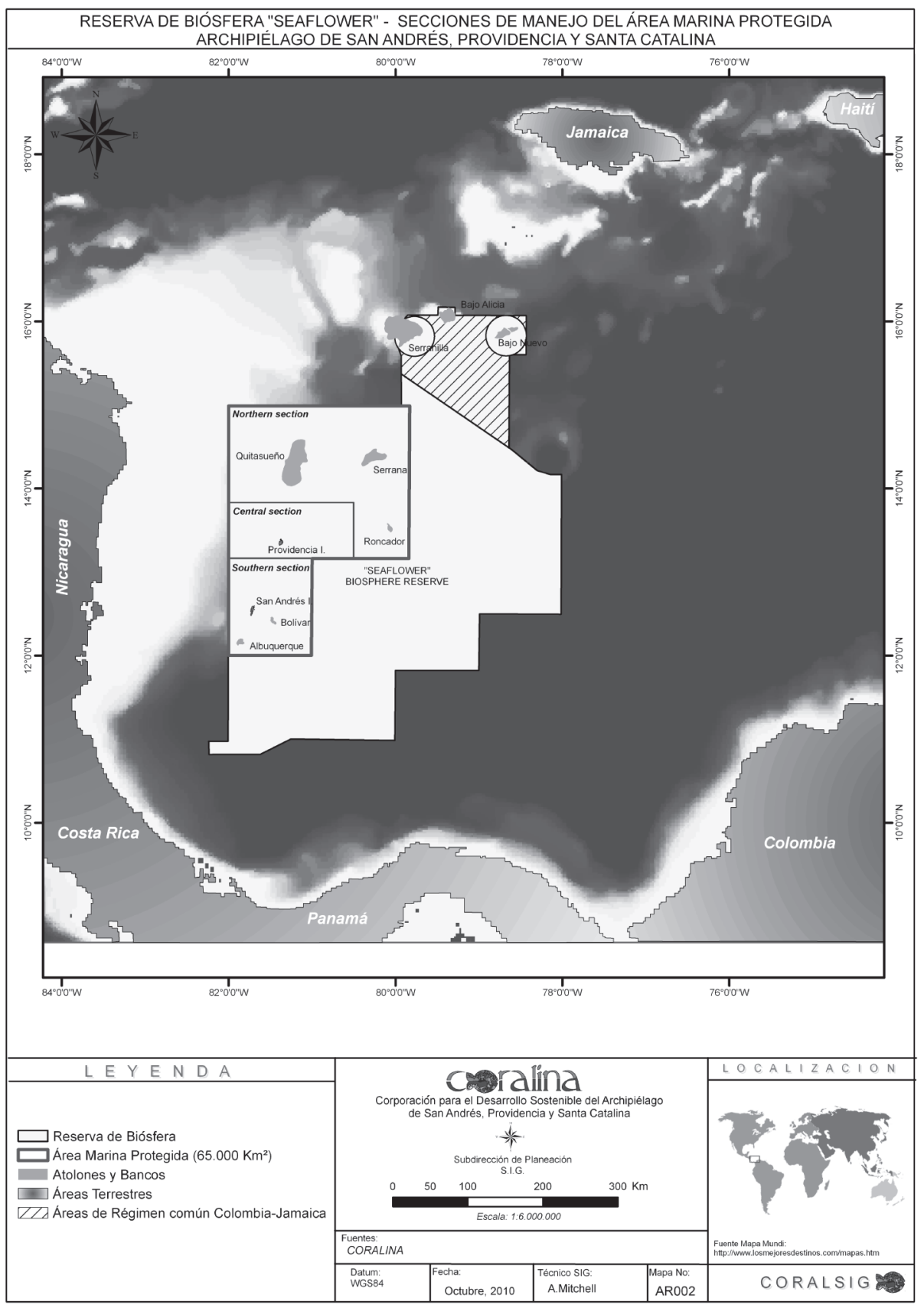

Fig. 1. Ubicación del Archipiélago de San Andrés, Providencia y Santa Catalina, Reserva de Biósfera Seaflower. Tomado de SIG Coralina (2011) y en el recuadro negro con líneas diagonales se señala el área de estudio

Fig. 1. Location of the Archipelago of San Andrés, Old Providence and Santa Catalina, Seaflower Biosphere Reserve. Taken from SIG Coralina (2011); study area marked by the black, hashed box 
estación fue muestreada por $20 \mathrm{~min}$., en un área de $20 \mathrm{~m}$ x $20 \mathrm{~m}$.

En el monitoreo se tuvieron en cuenta las especies de importancia comercial y ecológica que habitan en el Archipiélago, según el protocolo para monitoreo de peces de arrecife del manual de metodologías CARICOMP (2001) nivel II, basado a su vez en el programa Atlantic and Gulf Rapid Reef Assessment "AGRRA" (1999). La lista de especies fue escogida considerando los grupos taxonómicos que están siendo más impactados por la actividad pesquera y buscando información que pueda generar medidas adecuadas de protección y manejo.

Los datos fueron incorporados en bases de datos de EXCEL por estación e indicador medido. Con la metodología usada se obtuvieron datos de tipo cuantitativo, se estimaron los índices ecológicos de diversidad de Shannon y Simpson y de riqueza de Margalef con el software, PRIMER 6 (2005), para diversidad de peces. Adicionalmente, se elaboraron dendogramas de similaridades de Bray-Curtis, con el fin de definir similaridades de las estaciones muestreadas en cuanto a las abundancias de las especies observadas.

\section{RESULTADOS}

A continuación se describen las especies de peces con interés ecológico y económico registradas en Serranilla, Bajo Alicia y Bajo Nuevo (Cuadro 1).

\section{Serranilla}

En el muestreo de las 26 estaciones evaluadas en Serranilla, se observaron 65 especies con importancia ecológica y económica, donde los peces de tamaños pequeños como Acanthurus coeruleus (7\%, 421 ind. en 25 transectos de $400 \mathrm{~m}^{2}$ ) y Stegastes partitus obtuvieron las mayores abundancias $(6.5 \%, 378$ ind. en 25 transectos de $400 \mathrm{~m}^{2}$ ), seguidos de Mellichthys niger y Haemulon flavolineatum con un 6\% (367 y 363 ind. en 25 transectos de $400 \mathrm{~m}^{2}$ ). No obstante, otros peces de gran importancia comercial como pargos, roncos, chernas, ballestas y jureles, entre otros, tuvieron menores abundancias (entre 3 y 187 ind. en 25 transectos de $400 \mathrm{~m}^{2}$ ) (Fig. 2).

En los muestreos de abundancia de peces con importancia ecológica y económica discriminados por rangos de tallas en el banco Serranilla, en el rango entre 5 a $10 \mathrm{~cm}$ se observaron 51 especies y es el rango en el que se presentó el mayor número de individuos (3963 ind. en 25 transectos de $400 \mathrm{~m}^{2}$ ), donde $S$. partitus obtuvo las mayores abundancias $(8.8 \%$, 349 ind. en 18 transectos de $400 \mathrm{~m}^{2}$ ), seguidos de $A$. coeruleus $(7.4 \%, 292$ ind. en 21 transectos de $400 \mathrm{~m}^{2}$ ) y $S$. planifrons $\left(7 \%, 285\right.$ ind. en 10 transectos de $\left.400 \mathrm{~m}^{2}\right)$ (Fig. 3a). En el rango siguiente, de 11 a $20 \mathrm{~cm}$, se observaron 41 especies, donde $H$. flavolineatum ocupa la mayor representación de individuos (13.5\%, 154 ind. en 11 transectos de $400 \mathrm{~m}^{2}$ ), seguidos de $M$. niger $(12.6 \%, 143$ ind. en 11 transectos de $\left.400 \mathrm{~m}^{2}\right)$ y $A$. coeruleus $(9.7 \%, 110$ ind. en 13 transectos de $400 \mathrm{~m}^{2}$ ) (Fig. 3b). En el rango de 21 a $30 \mathrm{~cm}$ se observaron 23 especies, destacando a $M$. niger (32\%, 104 ind. en 10 transectos de $\left.400 \mathrm{~m}^{2}\right), B a$ listes vetula $(22 \%, 73$ ind. en 21 transectos de $\left.400 \mathrm{~m}^{2}\right)$ y $A$. chirurgus $(10 \%, 32$ ind. en 2 transectos de $400 \mathrm{~m}^{2}$ ) (Fig. 3c). En el rango de 31 a $40 \mathrm{~cm}$ se observaron 15 especies, siendo B. vetula el pez con mayor representación $(53 \%, 36$ ind. en 11 transectos de $400 \mathrm{~m}^{2}$ ), seguido de $M$. niger $\left(10 \%, 7\right.$ ind. en 2 transectos de $400 \mathrm{~m}^{2}$ ) (Fig. 3d). En el rango de 41 a $50 \mathrm{~cm}$ se observaron seis especies, donde $B$. vetula 
Cuadro 1. Listado de especies de peces con interés económico y ecológico observadas en los censos marinos de abundancia de peces realizados en Serranilla (SR), Bajo Alicia (BA) y Bajo Nuevo (BN)

Table 1. List of reef fish species with an ecological and economic value observed in all the marine census in Serranilla (SR), Alice Shoal (BA) and New Shoal (BN)

\begin{tabular}{|c|c|c|c|c|c|}
\hline Especies & Áreas & Especies & Áreas & Especies & Áreas \\
\hline Abudefduf saxatilis & SR-BN & Elagatis bipinnulata & $\mathrm{BN}$ & $\begin{array}{l}\text { Mulloidichthys } \\
\text { martinicus }\end{array}$ & BA-BN-SR \\
\hline Acanthurus bahianus & BA-BN-SR & Epinephelus guttatus & BA-BN & Mycteroperca tigris & $\mathrm{BN}$ \\
\hline Acanthurus chirurgus & BA-BN-SR & Equetus punctatus & $\mathrm{BN}$ & $\begin{array}{l}\text { Mycteroperca } \\
\text { venenosa }\end{array}$ & $\mathrm{BN}$ \\
\hline Acanthurus coeruleus & BA-BN-SR & $\begin{array}{l}\text { Gynglimostoma } \\
\text { cirratum }\end{array}$ & BA-BN-SR & Myripristis jacobus & BA-BN-SR \\
\hline Alphester affer & $\mathrm{BN}$ & Haemulon album & BA-BN-SR & Ocyurus chrysurus & $\mathrm{BN}$ \\
\hline Aluterus scriptus & $\mathrm{BN}$ & $\begin{array}{l}\text { Haemulon } \\
\text { aurolineatum }\end{array}$ & SR-BN & Paracanthias furcifer & $\mathrm{BN}$ \\
\hline Aulostomus maculatus & $\mathrm{BN}$ & $\begin{array}{l}\text { Haemulon } \\
\text { chrysargireum }\end{array}$ & $\mathrm{BN}$ & Pareques acuminatus & BA \\
\hline Balistes vetula & BA-BN-SR & $\begin{array}{l}\text { Haemulon } \\
\text { flavolineatum }\end{array}$ & BA-BN-SR & $\begin{array}{l}\text { Pomacanthus } \\
\text { arcuatus }\end{array}$ & $\mathrm{BN}$ \\
\hline Bodianus rufus & BA-BN-SR & Haemulon melanurum & BA-BN-SR & Pomacanthus paru & BA-BN \\
\hline Bothus lunatus & $\mathrm{BN}$ & Haemulon plumieri & BA-BN-SR & Priacanthus arenatus & $\mathrm{BN}$ \\
\hline Cantherhines macrocerus & $\mathrm{BA}$ & Halicoeres bivittatus & SR & $\begin{array}{l}\text { Pseudopeneus } \\
\text { maculatus }\end{array}$ & BA-BN-SR \\
\hline Cantherhines pullus & BA-BN & Halicoeres garnotti & SR-BN & Rypticus saponaceus & BA-BN \\
\hline Canthidermis sufflamen & BA-BN-SR & $\begin{array}{l}\text { Heteropriacanthus } \\
\text { cruentatus }\end{array}$ & $\mathrm{BN}$ & Scarus iseri & BA-BN-SR \\
\hline Canthigaster rostrata & BA-BN-SR & Holocanthus cilliaris & BA & Scarus taeniopterus & BA-BN-SR \\
\hline Caranx lugubris & BA-BN & Holocanthus tricolor & BA-BN-SR & Scarus vetula & SR-BN \\
\hline Caranx crysos & $\mathrm{BN}$ & $\begin{array}{l}\text { Holocentrus } \\
\text { adscensionis }\end{array}$ & BA-BN-SR & Serranus baldwini & BA \\
\hline Caranx ruber & BA-BN-SR & Hypoplectrus chlororus & $\mathrm{BN}$ & Serranus tigrinus & $\mathrm{BN}$ \\
\hline Cephalopholis cruentata & SR-BN & $\begin{array}{l}\text { Hypoplectrus } \\
\text { guttavarius }\end{array}$ & $\mathrm{BN}$ & $\begin{array}{l}\text { Sparisoma } \\
\text { aurofrenatum }\end{array}$ & BA-BN-SR \\
\hline Cephalopholis fulva & BA-BN-SR & Hypoplectrus nigricans & $\mathrm{BN}$ & $\begin{array}{l}\text { Sparisoma } \\
\text { chrysopterum }\end{array}$ & BA-BN-SR \\
\hline Chaetodon aculeatus & $\mathrm{BN}$ & $\begin{array}{l}\text { Hypoplectrus } \\
\text { providencianus }\end{array}$ & $\mathrm{BN}$ & Sparisoma rubripinne & BA-BN-SR \\
\hline Chaetodon capistratus & $\mathrm{BN}$ & Hypoplectrus puella & $\mathrm{BN}$ & Sparisoma viride & BA-BN-SR \\
\hline Chaetodon ocullatus & $\mathrm{BN}$ & Hypoplectrus unicolor & $\mathrm{BN}$ & Sphyraena barracuda & BA-BN-SR \\
\hline Chaetodon striatus & BA-BN-SR & Kyphosus sectatrix & $\mathrm{BN}$ & Stegastes adustus & SR-BN \\
\hline Chromis cianea & BA-BN-SR & Lactophris triqueter & SR-BN & Stegastes diencaeus & $\mathrm{BN}$ \\
\hline Chromis multilineata & BA-BN-SR & Lutjanus analis & SR-BN & Stegastes partitus & BA-BN-SR \\
\hline Clepticus parrae & SR & Malacanthus plumieri & BA-BN-SR & Stegastes planifrons & BA-BN-SR \\
\hline $\begin{array}{l}\text { Coryphopterus } \\
\text { glaucofraenum }\end{array}$ & SR & Malacoctenus boehlkei & SR & $\begin{array}{l}\text { Thalassoma } \\
\text { bifasciatum }\end{array}$ & SR \\
\hline Dasyatis americana & $\mathrm{BN}$ & $\begin{array}{l}\text { Malacoctenus } \\
\text { triangulatus }\end{array}$ & SR & Urobatis jamaicensis & $\mathrm{BN}$ \\
\hline Diodon holocanthus & $\mathrm{BN}$ & Mellichthys niger & BA-BN-SR & Xanthichthys ringens & BA-BN \\
\hline Diodon hystix & SR & $\begin{array}{l}\text { Microspathodon } \\
\text { chrysurus }\end{array}$ & BA-BN-SR & & \\
\hline
\end{tabular}




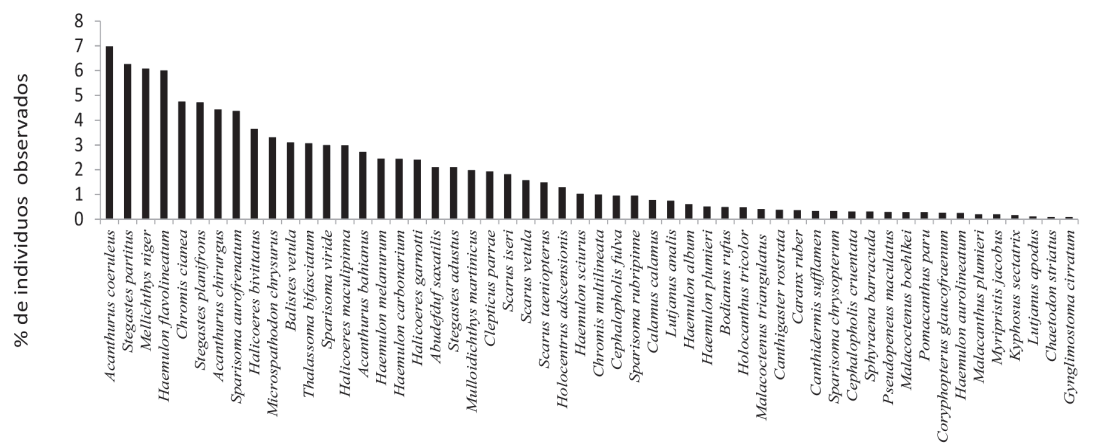

Fig. 2. Porcentaje de la abundancia de peces con importancia ecológica y económica registrados en Serranilla

Fig. 2. Abundance percentage of reef fish with an economic and ecological value observed in Serranilla

presentó el mayor porcentaje de abundancias $(61 \%, 14$ ind. en 4 transectos de 400 $\mathrm{m}^{2}$ ) (Fig. 3e) y en el rango de mayores de $50 \mathrm{~cm}$ solo se observaron a $S$. barracuda y los tiburones Gynglimostoma cirratum y Carcharhinus spp.
Al hacer el análisis de similaridad de Bray-Curtis con las estaciones censadas en Serranilla, se observa que las estaciones más parecidas son SrA13 y SrA15, con un 71.51\% de similaridad, seguidas por SrA14 y SrA16, con un $63.96 \%$ de similaridad (Fig. 4).

a) Rango de tallas de 5 a $10 \mathrm{~cm}$

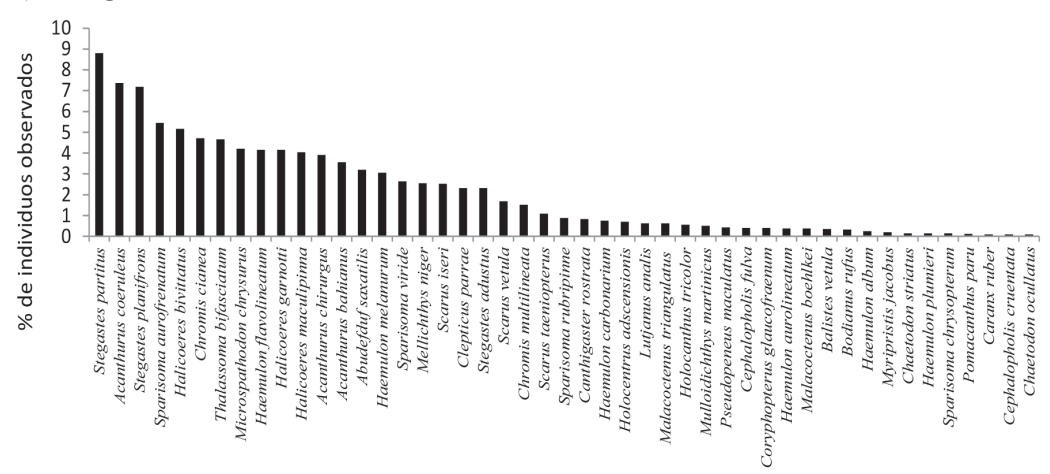

b) Rango de tallas de 11 a $20 \mathrm{~cm}$

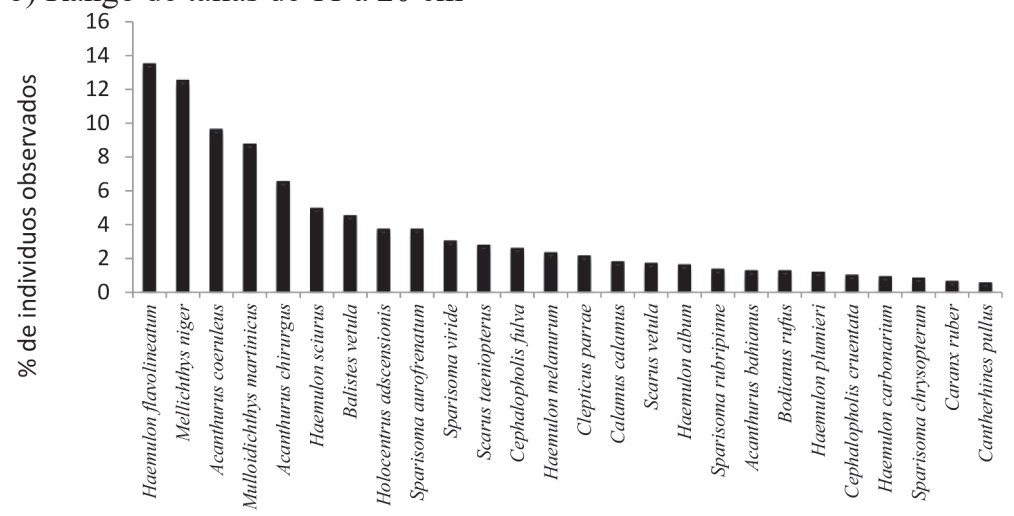


c) Rango de tallas de 21 a $30 \mathrm{~cm}$

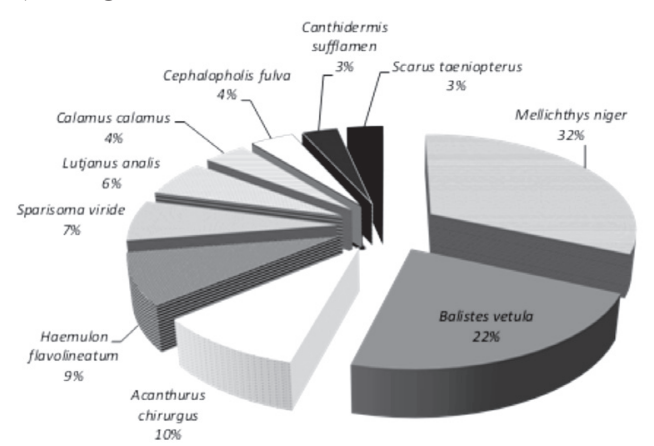

d) Rango de tallas de 31 a $40 \mathrm{~cm}$

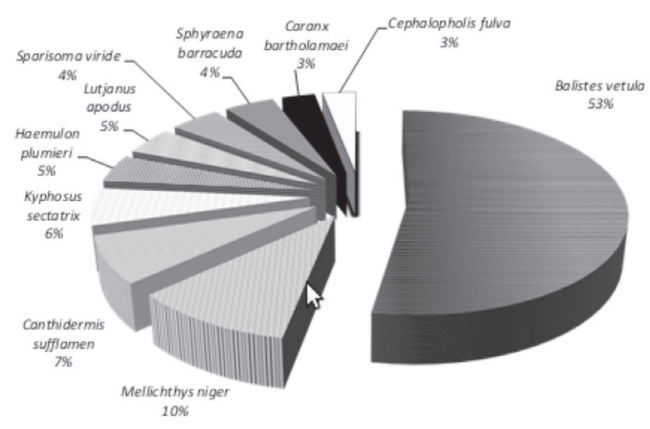

e) Rango de tallas de 41 a $50 \mathrm{~cm}$

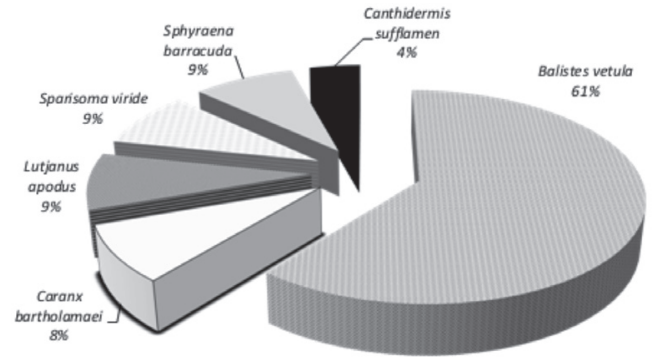

Fig. 3. Porcentaje de la abundancia de peces con importancia ecológica y económica discriminados por rango de tallas en Serranilla

Fig. 3. Abundance percentage of reef fish with economic and ecological value in Serranilla by size ranges

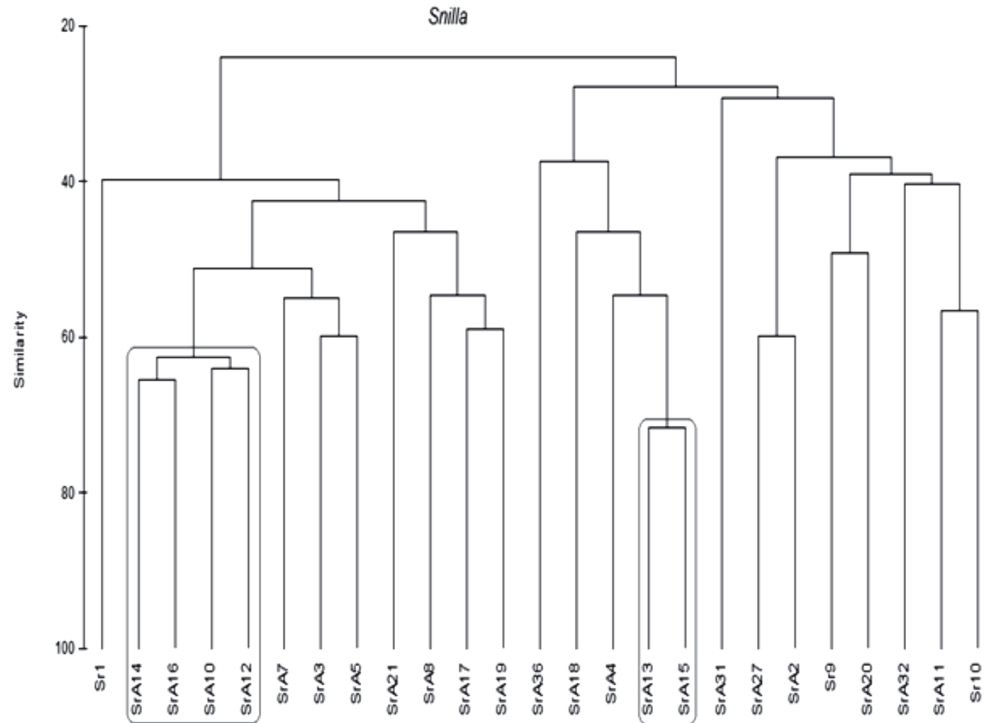

Fig. 4. Análisis de similaridad de Bray-Curtis de las estaciones muestreadas en Serranilla

Fig. 4. Dendogram based on Bray-Curtis Similarity Analysis for all stations sampled in Serranilla 
Al realizar el análisis de la diversidad de los peces con importancia ecológica y económica en Serranilla se puede detallar que existe una relación directa entre la diversidad y la riqueza en las estaciones más abundantes y con mayor número de especies, siendo las estaciones más diversas las número SrA10, 12, 14 y 16 y confirmando la relación entre las estaciones SrA10 y SrA12 (Cuadro 2), que se observa en el análisis de similaridad (Fig. 4).

\section{Bajo Alicia}

En el muestreo de las 14 estaciones evaluadas en Bajo Alicia, se observaron 47 especies con interés ecológico y económico (equivalentes a 3207 ind. en 14 transectos de $400 \mathrm{~m}^{2}$ ), donde $M$. niger (13.6\%) y C. ruber $(13.1 \%)$ obtuvieron las mayores abundancias (437 y 421 ind. en 14 transectos de $400 \mathrm{~m}^{2}$ ), seguidas de Acanthurus chirurgus $\left(12 \%, 381\right.$ ind. en 14 transectos de $\left.400 \mathrm{~m}^{2}\right)$, S. partitus $(9 \%, 275$ ind. en 14 transectos de $\left.400 \mathrm{~m}^{2}\right)$ y $B$. vetula $(5.7 \%, 213$ ind. en 14 transectos de $400 \mathrm{~m}^{2}$ ). No obstante, otros peces de gran importancia comercial como pargos, roncos, chernas, barracudas y peces loro, entre otros, tuvieron menores abundancias (entre 33 y 2 individuos) (Fig. 5).

Cuadro 2. Índices de diversidad y riqueza calculados para las estaciones evaluadas en Serranilla

Table 2. Diversity and richness indexes calculated for all sites sampled in Serranilla

\begin{tabular}{ccccccc|ccccccc}
\hline Estación & $\mathbf{S}$ & $\mathbf{N}$ & $\mathbf{d}$ & $\mathbf{J}^{\prime}$ & $\begin{array}{c}\mathbf{H}^{\prime} \\
\text { (Log 10) }\end{array}$ & 1-Lambda & Estación & $\mathbf{S}$ & $\mathbf{N}$ & $\mathbf{d}$ & $\mathbf{J}^{\prime}$ & $\begin{array}{c}\mathbf{H}^{\prime} \\
\text { (Log 10) }\end{array}$ & 1-Lambda' \\
\hline Sr1 & 28 & 513 & 4.3 & 0.81 & 1.17 & 0.90 & SrA2 & 10 & 30 & 3 & 0.90 & 0.90 & 0.88 \\
SrA10 & 30 & 466 & 4.7 & 0.89 & 1.32 & 0.94 & SrA4 & 16 & 96 & 9 & 0.89 & 1.07 & 0.91 \\
SrA12 & 29 & 354 & 4.8 & 0.90 & 1.31 & 0.94 & SrA36 & 15 & 477 & 2 & 0.71 & 0.84 & 0.82 \\
SrA14 & 18 & 44 & 2.9 & 0.96 & 1.20 & 0.94 & SrA31 & 14 & 233 & 2 & 0.62 & 0.71 & 0.69 \\
SrA16 & 31 & 512 & 4.8 & 0.88 & 1.32 & 0.94 & SrA32 & 13 & 76 & 3 & 0.83 & 0.92 & 0.86 \\
SrA17 & 19 & 216 & 3.3 & 0.89 & 1.13 & 0.91 & Sr9 & 18 & 85 & 4 & 0.92 & 1.16 & 0.93 \\
SrA19 & 27 & 341 & 4.5 & 0.92 & 1.32 & 0.95 & SrA11 & 18 & 113 & 4 & 0.72 & 0.91 & 0.78 \\
SrA21 & 16 & 127 & 3.1 & 0.85 & 1.02 & 0.88 & SrA13 & 23 & 106 & 5 & 0.88 & 1.20 & 0.92 \\
SrA3 & 26 & 292 & 4.4 & 0.89 & 1.26 & 0.93 & SrA15 & 19 & 117 & 4 & 0.89 & 1.14 & 0.92 \\
SrA5 & 20 & 353 & 3.2 & 0.88 & 1.14 & 0.92 & Sr10 & 18 & 151 & 3 & 0.77 & 0.97 & 0.84 \\
SrA7 & 24 & 443 & 3.8 & 0.90 & 1.24 & 0.93 & SrA18 & 25 & 179 & 5 & 0.82 & 1.15 & 0.91 \\
SrA8 & 27 & 330 & 4.5 & 0.90 & 1.29 & 0.94 & SrA20 & 11 & 57 & 2 & 0.87 & 0.91 & 0.85 \\
Sr27 & 12 & 52 & 2.8 & 0.93 & 1.00 & 0.90 & & & & & & & \\
\hline
\end{tabular}

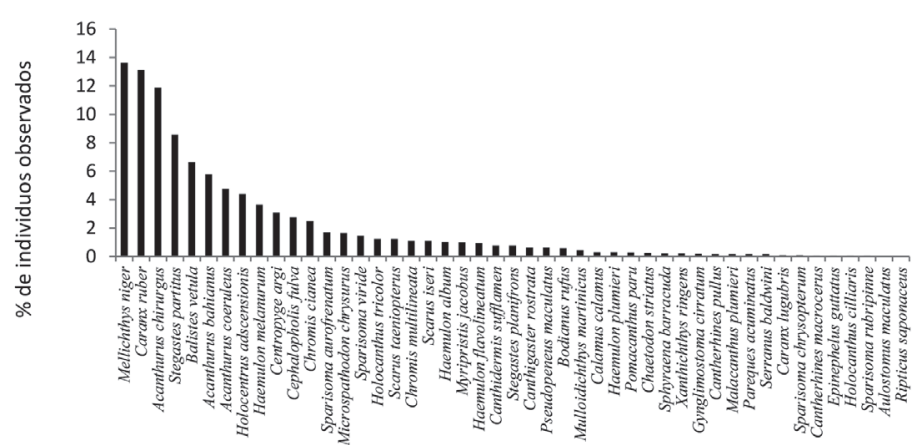

Fig. 5. Porcentaje de la abundancia de peces con importancia ecológica y económica registrados en Bajo Alicia

Fig. 5. Abundance percentage of reef fish with an economic and ecological value observed in Alice Shoal 
En los censos de abundancia de peces con importancia ecológica y económica discriminados por rangos de tallas, se observa que el rango entre 5 a $10 \mathrm{~cm}$ es el que mayor número de especies presentó, con 35 especies, donde $A$. chirurgus ( $15 \%$, 294 ind. en 13 transectos de $400 \mathrm{~m}^{2}$ ) y $S$. partitus $(14 \%, 275$ ind. en 12 transectos de $400 \mathrm{~m}^{2}$ ) fueron las más abundantes (Fig. 6a). En el rango de 11 a $20 \mathrm{~cm}$ se observaron 25 especies, destacando a $M$. niger $\left(27 \%, 249\right.$ ind. en 12 transectos de $\left.400 \mathrm{~m}^{2}\right)$ y C. ruber $(23 \%, 210$ ind. en 6 transectos de $400 \mathrm{~m}^{2}$ ) como las más abundantes (Fig. $6 b)$. En el rango de 21 a $30 \mathrm{~cm}$ se observaron 13 especies, destacando el dominio de los peces balistidos $(89 \%, 181$ ind. en 11

a) Rango de tallas de 5 a $10 \mathrm{~cm}$

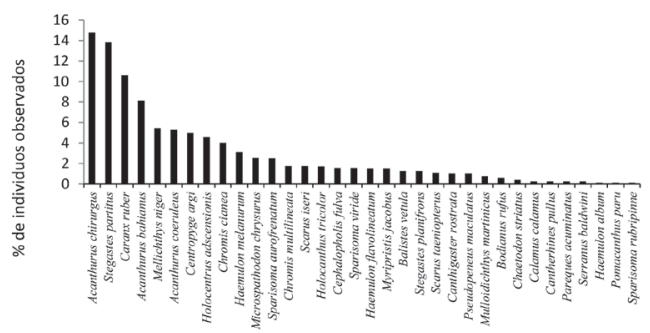

c) Rango de tallas de 21 a $30 \mathrm{~cm}$

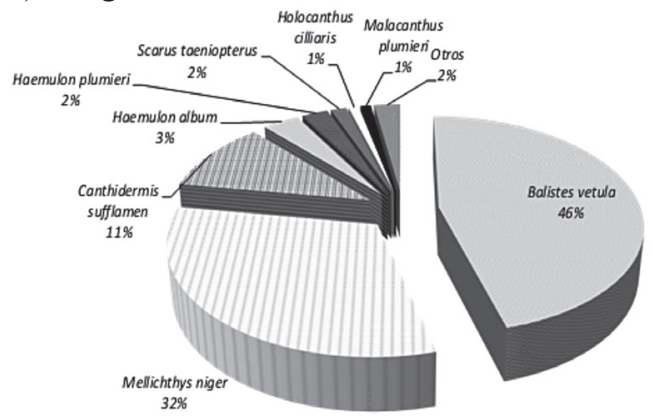

transectos de $\left.400 \mathrm{~m}^{2}\right)$, B. vetula $(46 \%, 94$ ind. en 11 transectos de $400 \mathrm{~m}^{2}$ ), M. niger $\left(32 \%, 65\right.$ ind. en 3 transectos de $\left.400 \mathrm{~m}^{2}\right) \mathrm{y}$ Canthidermis sufflamen $(11 \%, 22$ ind. en 3 transectos de $400 \mathrm{~m}^{2}$ ) (Fig. 6c). En el rango de 31 a $40 \mathrm{~cm}$ continuó el dominio de los peces balistidos, observando solo a $B$. vetula $\left(87 \%, 20\right.$ ind. en 5 transectos de $\left.400 \mathrm{~m}^{2}\right)$ y $C$. sufflamen $(13 \%, 3$ ind. en 2 transectos de $400 \mathrm{~m}^{2}$ ). En el rango de 41 a $50 \mathrm{~cm}$ solo se observó a Caranx lugubris, y en el rango de mayores de $50 \mathrm{~cm}$ se observaron solo cuatro especies, donde $S$. barracuda $\left(53 \%, 9\right.$ ind. en 5 transectos de $\left.400 \mathrm{~m}^{2}\right)$ y el tiburón nodriza, Gynglimostoma cirratum (35\%, 6 ind. en 5 transectos de $\left.400 \mathrm{~m}^{2}\right)$, fueron las más abundantes (Fig. 6d). b) Rango de tallas de 11 a $20 \mathrm{~cm}$

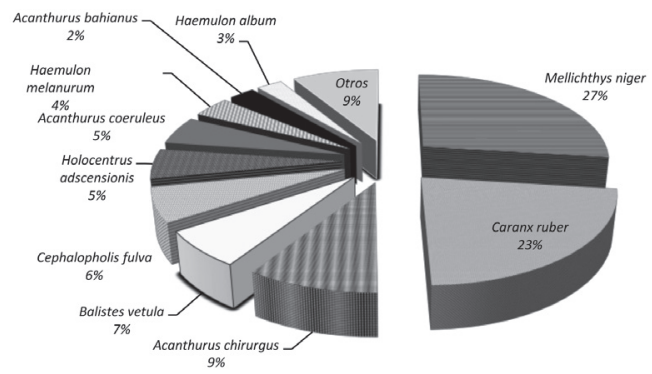

d) Rango de tallas mayores a $50 \mathrm{~cm}$

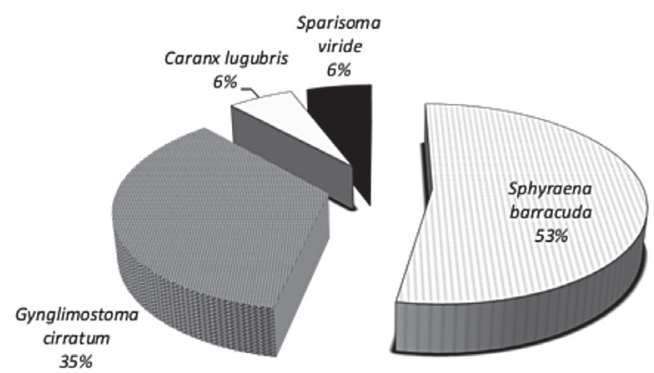

Fig. 6. Porcentaje de la abundancia de peces con importancia ecológica y económica discriminados por rango de tallas en Bajo Alicia

Fig. 6. Abundance percentage of reef fish with economic and ecological value in Alice Shoal by size ranges 
Al hacer el análisis de similaridad de Bray-Curtis de las estaciones censadas de Bajo Alicia, se observa que las estaciones más parecidas fueron BAA7 y BA7, con un $75.06 \%$ de similaridad, seguidas por BA1 y BAA6, con un $74.6 \%$ de similaridad $y$, por último, el de las estaciones Alice 5 y BAA4, con un $70.68 \%$ de similaridad (Fig. 7).
Al realizar el análisis de la diversidad de los peces con importancia ecológica y económica registrados en Bajo Alicia, se puede detallar que las estaciones más diversas no presentaron las más altas similaridades, pero sí las mayores riquezas, destacando las estaciones BA10, BA7, BA19 y Alice 8 como las más diversas (Cuadro 3 ).

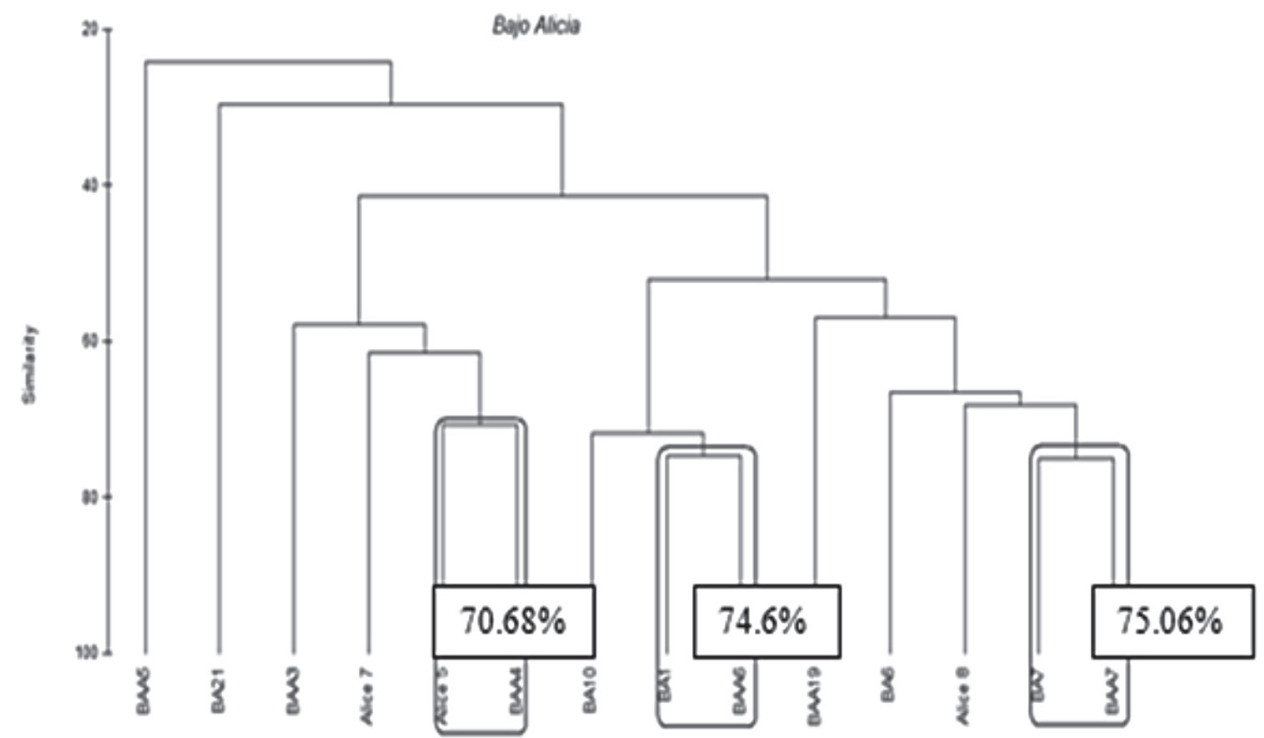

Fig. 7. Análisis de similaridad de Bray-Curtis de las estaciones muestreadas en Bajo Alicia

Fig. 7. Dendogram based on Bray-Curtis Similarity Analysis for all stations sampled in Alice Shoal

Cuadro 3. Índices de diversidad y riqueza calculados para las estaciones evaluadas en Bajo Alicia

Table 3. Diversity and richness indexes calculated for all stations sampled in Alice Shoal

\begin{tabular}{ccccccc}
\hline Estación & $\mathbf{S}$ & $\mathbf{N}$ & $\mathbf{d}$ & $\mathbf{J}^{\prime}$ & $\mathbf{H}^{\prime}(\mathbf{L o g}$ 10) & 1-Lambda' \\
\hline BA7 & 21 & 264 & 3.59 & 0.84 & 1.12 & 0.91 \\
BA6 & 22 & 122 & 4.37 & 0.84 & 1.12 & 0.89 \\
Alice 8 & 14 & 77 & 2.99 & 0.89 & 1.02 & 0.90 \\
BAA19 & 20 & 221 & 3.52 & 0.86 & 1.12 & 0.90 \\
BAA5 & 30 & 927 & 4.24 & 0.68 & 1.00 & 0.79 \\
BAA7 & 18 & 214 & 3.17 & 0.87 & 1.09 & 0.89 \\
BA21 & 8 & 28 & 2.10 & 0.72 & 0.64 & 0.69 \\
Alice 7 & 17 & 429 & 2.64 & 0.77 & 0.95 & 0.85 \\
Alice 5 & 14 & 265 & 2.33 & 0.67 & 0.77 & 0.75 \\
BAA3 & 12 & 58 & 2.71 & 0.83 & 0.90 & 0.86 \\
BAA4 & 10 & 136 & 1.83 & 0.73 & 0.73 & 0.74 \\
BA1 & 17 & 155 & 3.17 & 0.81 & 1.00 & 0.87 \\
BA10 & 22 & 124 & 4.36 & 0.86 & 1.16 & 0.92 \\
BAA6 & 19 & 187 & 3.44 & 0.80 & 1.03 & 0.88 \\
\hline
\end{tabular}




\section{Bajo Nuevo}

En el muestreo de las 42 estaciones evaluadas en Bajo Nuevo, se observó un total de 78 especies con importancia económica y ecológica (7206 ind. en 38 transectos de $\left.400 \mathrm{~m}^{2}\right)$, donde peces como $A$. coeruleus $(13 \%, 886$ ind. en 38 transectos de $\left.400 \mathrm{~m}^{2}\right)$ y $S$. partitus $(7 \%, 519$ ind. en 38 transectos de $400 \mathrm{~m}^{2}$ ) obtuvieron las mayores abundancias, seguidos de una gran variedad de peces arrecifales como peces mariposa (Chaetodon spp.), cirujanos, cofres, etc., mientras que otros de gran importancia comercial como pargos, roncos, chernas, barracudas, loros, entre otros, y algunos condrictios como las rayas, tuvieron una baja abundancia (Fig. 8). Por su parte, algunos tiburones ( $G$. cirratum) mostraron mayor abundancia en esta zona, algo incluso mayor a lo registrado en otras áreas del Archipiélago, lo cual fue confirmado en los censos de riqueza íctica.
En los censos de abundancia de peces con interés ecológico y económico discriminados por rangos de tallas en Bajo Nuevo, se observa que el rango entre 5 a $10 \mathrm{~cm}$ es el que mayor cantidad de especies presentó (63 especies), donde $A$. coeruleus $(13.5 \%, 823$ ind. en 34 transectos de $400 \mathrm{~m}^{2}$ ) y $S$. partitus $(9 \%, 524$ ind. en 30 transectos de $400 \mathrm{~m}^{2}$ ) fueron las más abundantes (Fig. 9a). En el rango siguiente, de 11 a $20 \mathrm{~cm}$, se observó un total de 37 especies, destacando a $M$. niger $(14 \%, 92$ ind. en 8 transectos de $\left.400 \mathrm{~m}^{2}\right)$ y $\mathrm{Mu}$ lloidichthys martinicus (11\%, 75 ind. en 8 transectos de $400 \mathrm{~m}^{2}$ ) como las más abundantes (Fig. 9b). En el rango de 21 a $30 \mathrm{~cm}$ se observaron 16 especies, destacando el dominio de los peces balistidos, B. vetula $(32 \%, 67$ ind. en 18 transectos de $\left.400 \mathrm{~m}^{2}\right)$ y $M$. niger $(12 \%, 25$ ind. en 2 transectos de $\left.400 \mathrm{~m}^{2}\right)$, y también a Haemulon album $(14 \%, 29$ ind.

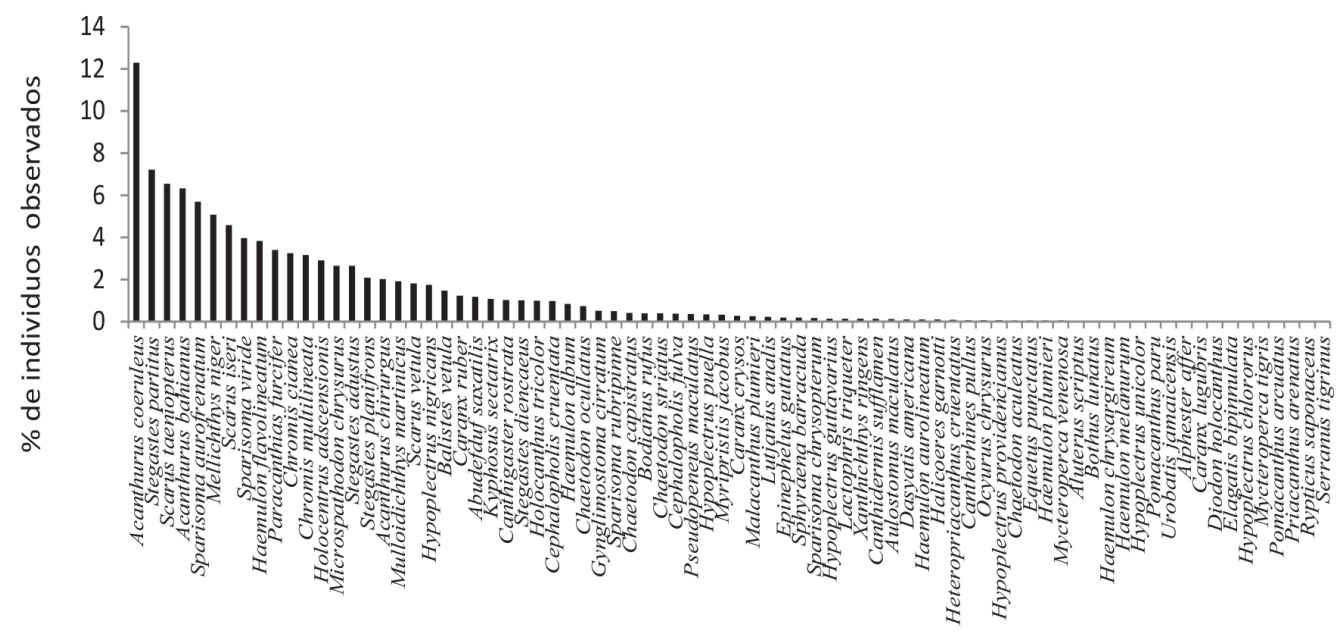

Fig. 8. Porcentaje de la abundancia de peces con importancia ecológica y económica registrados en Bajo Nuevo

Fig. 8. Abundance percentage of reef fish with an economic and ecological value observed in New Shoal 
en 4 transectos de $400 \mathrm{~m}^{2}$ ) (Fig. 9c). En el rango de 31 a $40 \mathrm{~cm}$ continuó el dominio de $H$. album $(56 \%, 22$ ind. en 2 transectos de $\left.400 \mathrm{~m}^{2}\right)$ y B. vetula $(33 \%$, 13 ind. en 6 transectos de $400 \mathrm{~m}^{2}$ ). En el rango de 41 a $50 \mathrm{~cm}$ se continuaron observando las altas abundancias de los balistidos $(63 \%)$, representados por C. sufflamen $\left(42 \%, 8\right.$ ind. en 2 transectos de $\left.400 \mathrm{~m}^{2}\right)$ y $B$. vetula $(21 \%, 4$ ind. en 2 transectos de 400 $\mathrm{m}^{2}$ ) (Fig. 9d), y en el rango de mayores de $50 \mathrm{~cm}$ se observaron mayores abundancias

a) Rango de tallas de 5 a $10 \mathrm{~cm}$

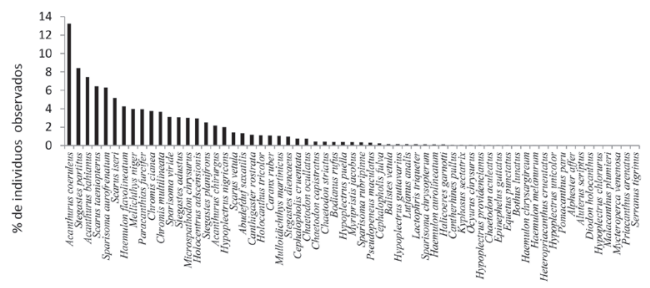

c) Rango de tallas de 21 a $30 \mathrm{~cm}$

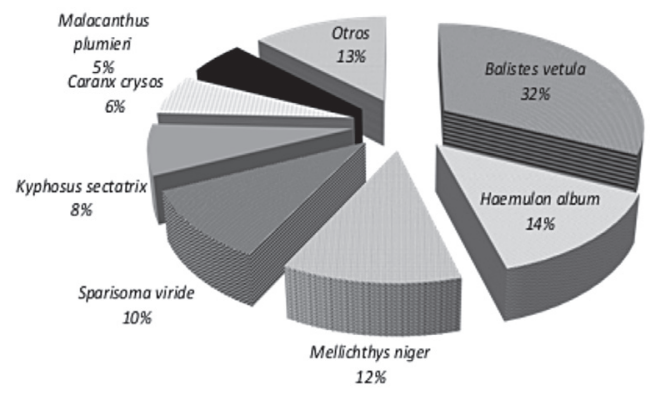

e) Rango de tallas mayores a $50 \mathrm{~cm}$

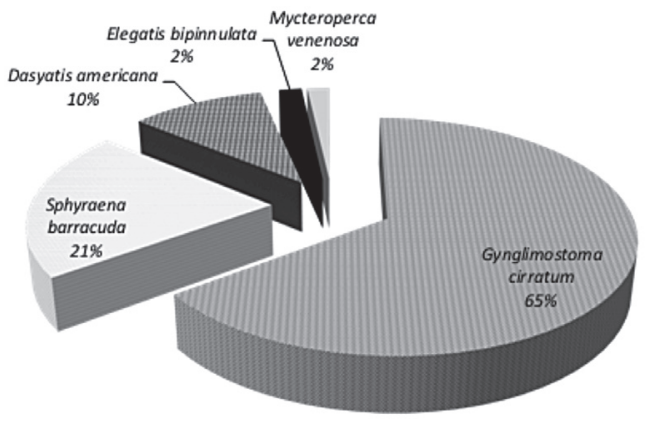

de condrictios $(75 \%)$, destacando al tiburón nodriza $G$. cirratum $(65 \%, 37$ ind. en 20 transectos de $400 \mathrm{~m}^{2}$ ) y la raya Dasyatis americana $(10 \%, 6$ ind. en 5 transectos de $400 \mathrm{~m}^{2}$ ), estos acompañados del pez $S . b a$ rracuda $(21 \%, 12$ ind. en 7 transectos de $400 \mathrm{~m}^{2}$ ) (Fig. 9e). En Bajo Nuevo se observa que las densidades de $G$. cirratum son muy superiores a las registradas para las otras áreas del Archipiélago (hasta de 5-9 individuos en la mayoría de las estaciones cercanas a la barrera arrecifal).

b) Rango de tallas de 11 a $20 \mathrm{~cm}$

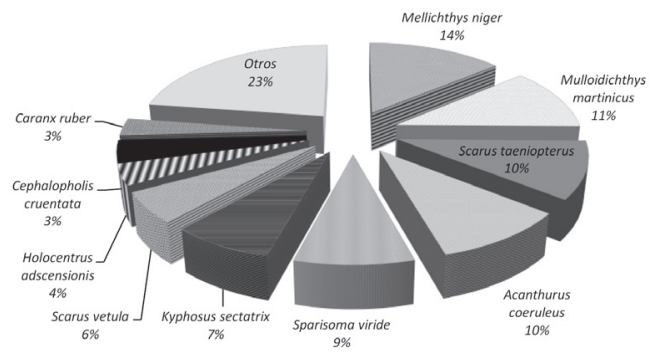

d) Rango de tallas de 41 a $50 \mathrm{~cm}$

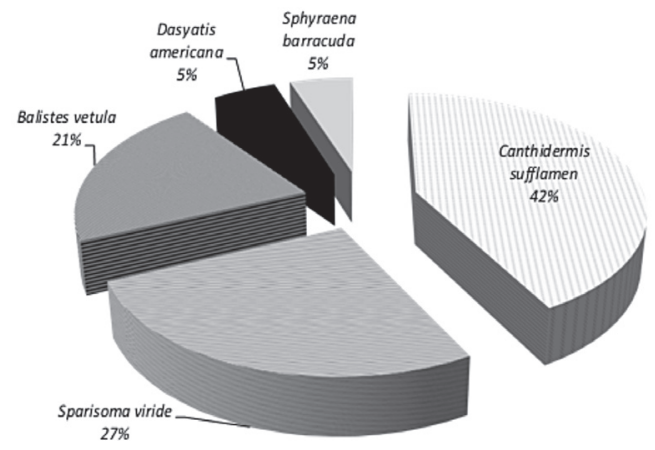

Fig. 9. Porcentaje de la abundancia de peces con importancia ecológica y económica discriminados por rango de tallas en Bajo Nuevo Fig. 9. Abundance percentage of reef fish with economic and ecological value in New Shoal by size ranges 
Al hacer el análisis de similaridad de Bray-Curtis de estaciones censadas en Bajo Nuevo, se observa que las estaciones más parecidas fueron $\mathrm{BN} 10$ y $\mathrm{BN} 8$, con un $73.52 \%$ de similaridad, seguidas por BN51 y BN39, con un $71.08 \%$ de similaridad $\mathrm{y}$, en contraste, las estaciones que presentaron menor similaridad fueron las estaciones BN1 y BN12, con un 30\% de similaridad (Fig. 10).
Al realizar el análisis de la diversidad de los peces con importancia ecológica y económica registrados en Bajo Nuevo se puede detallar que las estaciones más diversas no presentaron las más altas similaridades, pero sí las mayores riquezas de las especies con importancia ecológica y económica, destacando las estaciones BN10, BN3, BN28, BN68, BN49, BN66 y BN6 como las más diversas (Cuadro 4).

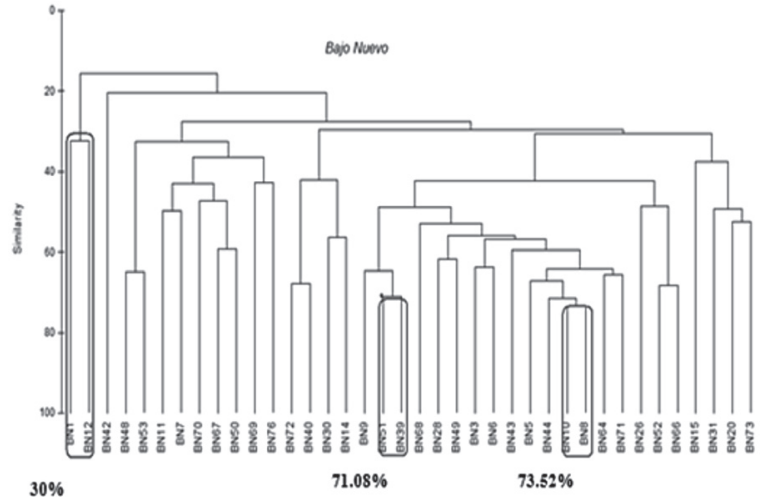

Fig. 10. Análisis de similaridad de Bray-Curtis de las estaciones muestreadas en Bajo Nuevo

Fig. 10. Dendogram based on BrayCurtis Similarity Analysis for all stations sampled in New Shoal

Cuadro 4. Índices de diversidad y riqueza calculados para las estaciones muestreadas en Bajo Nuevo

Table 4. Diversity and richness indexes calculated for all stations sampled in New shoal

\begin{tabular}{cccccccccccccc}
\hline Estación & $\mathbf{S}$ & $\mathbf{N}$ & $\mathbf{d}$ & $\mathbf{J}^{\prime}$ & $\begin{array}{c}\mathbf{H}^{\prime} \\
\text { (Log 10) }\end{array}$ & 1-Lambda' & Estación & $\mathbf{S}$ & $\mathbf{N}$ & $\mathbf{d}$ & $\mathbf{J}^{\prime}$ & $\begin{array}{c}\mathbf{H}^{\prime} \\
\text { (Log 10) }\end{array}$ & 1-Lambda' \\
\hline BN64 & 22 & 310 & 3.66 & 0.89 & 1.19 & 0.92 & BN39 & 10 & 151 & 1.79 & 0.86 & 0.86 & 0.83 \\
BN26 & 24 & 360 & 3.91 & 0.79 & 1.09 & 0.88 & BN66 & 24 & 251 & 4.16 & 0.87 & 1.20 & 0.93 \\
BN30 & 19 & 179 & 3.47 & 0.81 & 1.04 & 0.87 & BN42 & 19 & 283 & 3.19 & 0.45 & 0.57 & 0.49 \\
BN31 & 15 & 100 & 3.04 & 0.91 & 1.07 & 0.91 & BN67 & 11 & 49 & 2.57 & 0.86 & 0.90 & 0.86 \\
BN52 & 17 & 169 & 3.12 & 0.86 & 1.06 & 0.89 & BN11 & 29 & 151 & 5.58 & 0.77 & 1.13 & 0.88 \\
BN28 & 26 & 211 & 4.67 & 0.91 & 1.29 & 0.94 & BN7 & 25 & 257 & 4.33 & 0.66 & 0.92 & 0.78 \\
BN9 & 24 & 199 & 4.35 & 0.86 & 1.19 & 0.92 & BN12 & 16 & 349 & 2.56 & 0.63 & 0.76 & 0.76 \\
BN51 & 19 & 187 & 3.44 & 0.83 & 1.06 & 0.88 & BN72 & 20 & 472 & 3.09 & 0.58 & 0.75 & 0.72 \\
BN5 & 18 & 165 & 3.33 & 0.90 & 1.13 & 0.92 & BN14 & 10 & 40 & 2.44 & 0.86 & 0.86 & 0.85 \\
BN44 & 21 & 180 & 3.85 & 0.86 & 1.13 & 0.91 & BN40 & 16 & 188 & 2.86 & 0.53 & 0.64 & 0.58 \\
BN10 & 26 & 190 & 4.76 & 0.87 & 1.24 & 0.93 & BN15 & 8 & 26 & 2.15 & 0.90 & 0.81 & 0.86 \\
BN3 & 28 & 246 & 4.90 & 0.89 & 1.29 & 0.94 & BN48 & 19 & 221 & 3.33 & 0.67 & 0.86 & 0.76 \\
BN1 & 17 & 230 & 2.94 & 0.87 & 1.07 & 0.90 & BN69 & 22 & 140 & 4.25 & 0.80 & 1.07 & 0.88 \\
BN6 & 21 & 151 & 3.99 & 0.93 & 1.23 & 0.94 & BN70 & 20 & 134 & 3.88 & 0.87 & 1.13 & 0.90 \\
BN8 & 16 & 149 & 3.00 & 0.93 & 1.12 & 0.92 & BN20 & 20 & 93 & 4.19 & 0.84 & 1.10 & 0.90 \\
BN68 & 22 & 170 & 4.09 & 0.90 & 1.21 & 0.93 & BN73 & 18 & 88 & 3.80 & 0.86 & 1.08 & 0.90 \\
BN49 & 23 & 163 & 4.32 & 0.90 & 1.23 & 0.93 & BN53 & 20 & 190 & 3.62 & 0.65 & 0.85 & 0.71 \\
BN43 & 16 & 175 & 2.90 & 0.90 & 1.08 & 0.91 & BN76 & 27 & 327 & 4.49 & 0.81 & 1.16 & 0.91 \\
BN71 & 23 & 166 & 4.30 & 0.83 & 1.17 & 0.92 & BN50 & 17 & 96 & 3.51 & 0.89 & 1.10 & 0.91 \\
\hline
\end{tabular}




\section{DISCUSIÓN}

En las áreas muestreadas dentro del AMP Seaflower, se observaron que las comunidades de peces coralinos, en las estaciones monitoreadas, presentan una diversidad (\# de individuos) de baja a media de individuos, siendo estas muy similares entre las zonas norte y externa, a excepción de Bajo Alicia; estas diferencias en estas diversidades pueden deberse a efectos del muestreo, a las extensiones de arrecifes presentes en cada una de las áreas, siendo estas de grandes extensiones en los cayos del norte y externos, a excepción de Bajo Alicia que no presenta una barrera arrecifal extensa ni áreas someras, además fue una de las áreas donde menor cantidad de estaciones fueron monitoreadas.

Entre los resultados obtenidos es importante destacar las altas abundancias de los peces arrecifales como los cirujanos (en especial $A$. coeruleus) y damiselas (género Stegastes) (Fig. 11) en las tallas inferiores de los $20 \mathrm{~cm}$ y destacando a los peces loro, roncos, jureles y balistidos en las tallas superiores a $20 \mathrm{~cm}$. Peces de valor comercial (pc) como algunos balistidos
(B. vetula y C. sufflamen), jureles (C. ruber), loros (Scarus coeruleus, S. coelestinus, Sparisoma rubripinne y $S$. viride), pargos, barracudas, chernas y meros fueron poco abundantes, observados en las estaciones de mayor profundidad y fueron abundantes en un 35\% en Bajo Alicia; los otros peces arrecifales fueron más abundantes en Bajo Nuevo y Serranilla, donde se observaron porcentajes de abundancia entre el 80 y $90 \%$ (Fig. 12). Estas bajas abundancias de peces de interés comercial en estas áreas pueden deberse, según Nagelkerken et al. $(2000,2001)$, a una ausencia (o rara presencia) de hábitats como pastos marinos y manglares esenciales para las etapas juveniles de estas especies en las áreas estudiadas, lo cual se confirma al comparar estas abundancias con los resultados obtenidos en las AMP's centro y sur del Archipiélago, donde los pargos, chernas y meros fueron más abundantes, como resultado de la presencia de hábitats claves y la fuerte actividad de pesca presente también en la zona externa.

En adición a los resultados obtenidos y como lo expresan Dahlgren et al. (2003), es muy importante resaltar que las altas

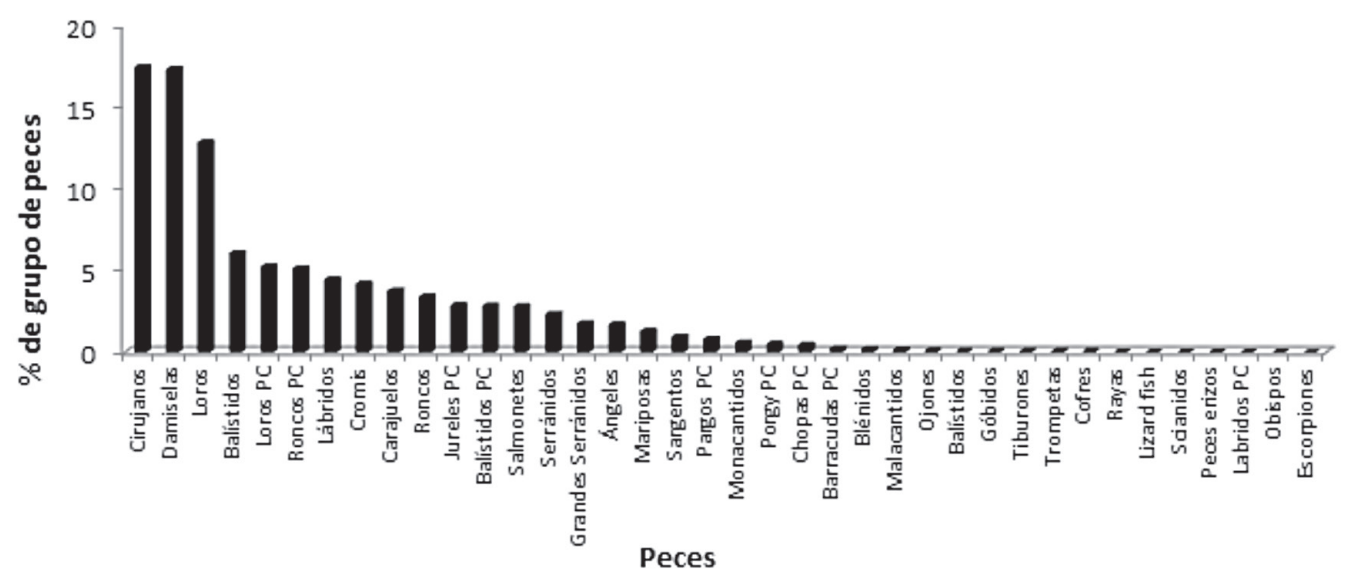

Fig. 11. Abundancia de individuos (\%) observados en las áreas de monitoreo

Fig. 11. Abundance of reef fish (\%) observed in sampled areas 


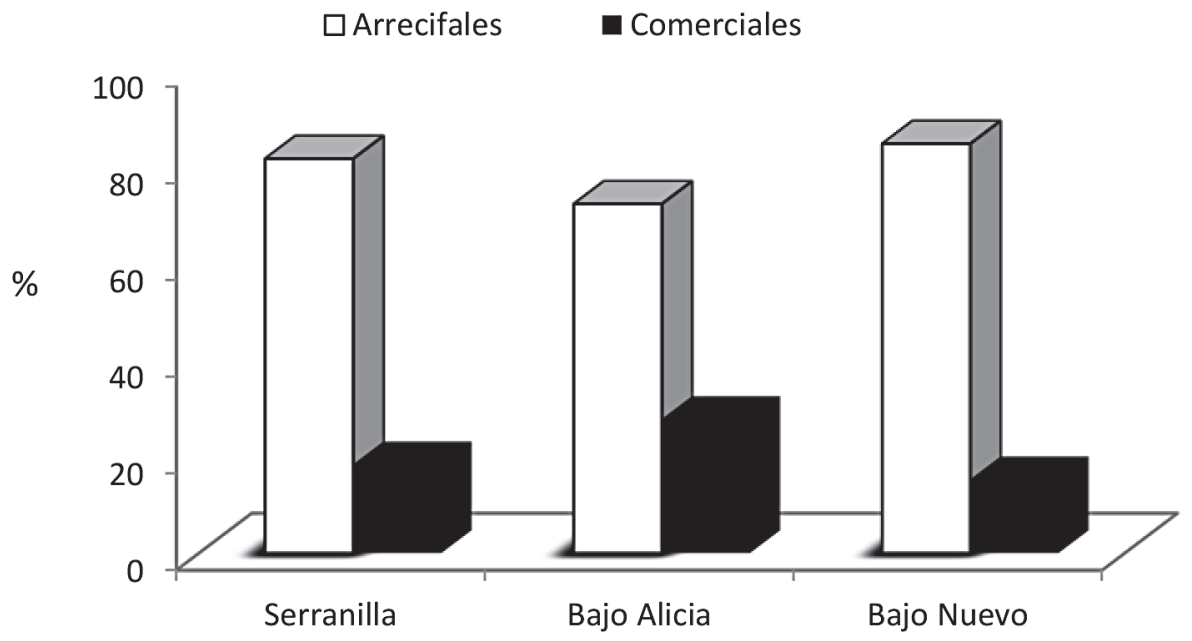

Fig. 12. Porcentaje de abundancia de individuos observados en las áreas de monitoreo según el grupo a que pertenecen

Fig. 12. Abundance percentage of reef fish observed in sampled areas by size groups

abundancias de peces de los niveles tróficos bajos como los herbívoros (cirujanos, doncellas y algunos peces loro), pueden ser una consecuencia de las bajas poblaciones de piscívoros como barracudas, pargos, chernas, meros y tiburones (ausencia de niveles altos), ocasionadas por la fuerte actividad de pesca presente. No obstante, las altas abundancias de herbívoros pueden ser beneficiosas para la salud del coral, manteniendo controlado el crecimiento de las algas. Otras especies con bajas densidades, como los peces ángeles y mariposas, pueden deberse a las bajas densidades, de esponjas en las áreas. Como conclusión de los resultados obtenidos y como función de la RB Seaflower, se deben aplicar medidas para la protección de las especies de importancia pesquera y de toda la diversidad de peces arrecifales presentes en el Archipiélago.

\section{AGRADECIMIENTOS}

Agradecimientos muy especiales a todos los que de alguna manera apoyaron el desarrollo de este estudio. A la Corporación para el Desarrollo Sostenible del Archipiélago de San Andrés, Providencia y Santa Catalina (CORALINA) y todo su personal. A la Gobernación Departamental, por intermedio de la Secretaría de Agricultura y Pesca del Departamento. Al capitán y la tripulación de la embarcación Captain "S", expedicionarios y colegas que nos acompañaron en la expedición científica. 


\section{BIBLIOGRAFÍA}

AGRRA. (1999). The AGRRA Rapid Assessment Protocol. Mesoamerican Reef System Workshop. Miami, EE.UU.: MGG-RSMAS, University of Miami.

CARICOMP. (2001). CARICOMP Methods Manual - Level I. CARICOMP Management Center, University of the West Indies, Mona, Kingston, Jamaica and Florida. Institute of Oceanography, University of South Florida, St. Petersburg Florida, U.S.A., Mona, Kingston, Jamaica.

Clarke, K. R. \& Gorley, R. N. (2005). Plymouth Routines In Multivariate Ecological Research. Lutton, United Kindom. PRIMER-E Ltd.

Corporación Regional para el Desarrollo Sostenible del Archipiélago de San Andrés, Providencia y Santa Catalina "CORALINA". (2010). Expedición cientifica de recolección de datos biológicos en Serrana, Roncador y primera aproximación al conocimiento de las comunidades coralinas e ícticas de los complejos arrecifales de Serranilla, Bajo Alicia y Bajo Nuevo - Colombia, Sección norte de la reserva de Biósfera Seaflower, Caribe occidental. San Andrés island. Colombia: CORALINA.

Corporación Regional para el Desarrollo Sostenible del Archipiélago de San Andrés, Providencia y Santa Catalina "CORALINA". (2009). Informe de los monitoreos de abundancia y diversidad de peces e invertebrados en las Evaluaciones Rápidas de Arrecifes en San Andrés y Providencia. San Andrés island. Colombia: CORALINA.

Dahlgren, C., Arboleda, E., Buch, K. L., Caldas, J. P., Posada, S. \& Prada, M. (2003). Characterization of reeffish diversity, community structure, distribution and abundance on three Southwestern Caribbean atolls: Quitasueño, Serrana and Roncador Banks (Seaflower Biosphere Reserve), Archipelago of San Andrés and Old Providence, Colombia. San Andrés island, Colombia: Corporation for the sustainable development of the San Andrés, Old Providence and Santa Catalina Archipelago (CORALINA).

Heinemann, D., Appeldoorn, R., Dahlgreen, C., Herrón, P., Prada, M. \& Sánchez, J. (2004). Rapid ecological assessment of the northern banks of the Archipelago of San Andrés and Old Providence. San Andrés island. Colombia: Joint Ocean Concervacy-CORALINA.

Herrón, P. (2004). Informe final de las tendencias de cambio en la estructura y estado de los arrecifes coralinos de San Andrés, Providencia y Santa Catalina: Programa de Monitoreo de CORALINA durante el periodo 1998-2002. San Andrés Isla, Colombia: CORALINA.

Instituto Geográfico Agustín Codazzi. "IGAC", Subdirección de Investigación y Divulgación Geográfica. (1986). Aspectos geográficos de San Andrés y Providencia. Bogotá D. C., Colombia: Ministerio de Hacienda y Crédito Público, Instituto Geográfico Agustín Codazzi, Subdirección de Investigación y Divulgación Geográfica.

Nagelkerken, I., van der Velde, G., Gorissen, M. W., Meijer, G. J., Van't Hof, T. $\&$ den Hartog, C. (2000). Importance of mangroves, seagrass beds and the shallow coral reefs as a nursery for important coral reef fishes, using a visual census technique. Estuar. Coast. Shelf. Sci., 51, 31-44.

Nagelkerken, I., Kleijnen, S., Klop, T., van den Brand, R. A. C. J., Cocheret 
de la Morini-ère, E. \& van der Velde, G. (2001). Dependence of Caribbean reef fishes on mangroves and seagrass beds as nursery habitats: a comparison of fish faunas between bays with and without mangroves/seagrass beds. Mar. Ecol. Progr. Ser., 214, 225-235.

Posada, O., Rozo, D. M., Bolaños, J. \& Zamora, A. (2009). Informe del estado de los ambientes y recursos marinos y costeros en Colombia: Año 2008. L. F. Santiago (Ed.), Marco Geográfico (pp. 19-26). Bogotá, Colombia: INVEMAR. PRIMER 6. (2005). Primer-E Ltd. 3 Meadow View, version 6 http://www.primer-e.com/primer.htm. Lutton, United Kingdom, PRIMER-E Ltd.

Rodríguez-Ramírez, A., Garzón-Ferreira, J., Bejarano-Chavarro, S., Navas-Camacho, R., Reyes-Nivia, C., Duque, G., Orozco, C., Zapata, F. \& Herrera, O. (2005). Informe del estado de los ambientes marinos y costeros en
Colombia: Año 2004. En INVEMAR (Ed.), Estado de los arrecifes coralinos en Colombia (pp. 77-114). Santa Marta, Colombia: Serie Publicaciones Periódicas 9.

Sánchez, J. A., Pizarro, V., Acosta, A. R., Castillo, P. A., Herron, P., Martínez, J. C., Montoya, P. \& Orozco, C. (2005). Evaluating coral reef benthic communities in remote Caribbean atolls (Quitasueño, Serrana, and Roncador Banks) to recommend marine-protected areas for the Seaflower Biosphere Reserve. Atoll Res. Bull., 531, 1-66.

Sistema de Información Geográfica de la Corporación Regional para el Desarrollo Sostenible del Archipiélago de San Andrés, Providencia y Santa Catalina (SIG-CORALINA). (2011). Ubicación Geográfica del Archipiélago de San Andrés, Providencia y Santa Catalina. San Andrés isla, Colombia: CORALINA. 